\title{
Modeling of electro-conductive properties of woven structure based on mixing model
}

\author{
Magdalena Tokarska ${ }^{1, *}$ \\ ${ }^{1}$ Institute of Architecture of Textiles, Lodz University of Technology, Poland \\ ${ }^{*}$ Corresponding author E-mail address: magdalena.tokarska@p.lodz.pl
}

\section{INFO}

CDAPT, ISSN: 2701-939X

Peer reviewed article

2020, Vol.1, Nr. 3, pp. 12-19

DOI:10.25367/cdatp.2020.1.p12-19

Received: 29 April 2020

Accepted: 21 Mai 2020

Available online: 22 September 2020

\section{Keywords}

woven structure,

electrical conductivity,

composite,

mixing model,

generalized Archie's law,

connectivity,

connectedness

\begin{abstract}
The main purpose of the research is to model the electro-conductive properties of the woven structure based on the mixing model. The generalized Archie's law for analysis of the woven structure and its conductivity was chosen. The generalized Archie's law can be applied to objects containing pores filled with phases. The phases are pathways enabling conduction. The remaining part is a nonconducting matrix. The model of the composite was transferred to a woven structure. Electro-conductive components of woven structure i.e. strips and contacts of strips were treated as conducting phases in the structure. The remaining part is pore space corresponds to the matrix. Based on generalized Archie's law, the connectivity as being a measure of how the components of the whole structure are arranged, and the connectedness of a given phase as being a measure of the availability of pathways for conduction through that phase was determined for all structures. It was found that the connectivity of the strips phase is higher than the connectivity of the contacts of strips phase. It means that the strips phase (in terms of their quantity) has a greater effect on the conductivity of the woven structure than the contacts of strips phase. A decrease of the connectedness of strips and contacts of strips phases (in terms of their quality) can be obtained by adding another component to the woven structure which will reduce the conductivity of the structure.
\end{abstract}

(c) 2020 The authors. Published by CDAPT.

This is an open access article under the CC BY-NC-ND license https://creativecommons.org/licenses/ peer-review under responsibility of the scientific committee of the CDAPT. 


\section{Introduction}

Textile materials are fundamental components of our everyday lives. Nowadays, consumers are becoming more and more demanding. Especially, they expect clothing to contain many functionalities. By combining traditional textiles and new fabrication technologies, we can achieve new functionalities [1-3]. As a result, the area of applications of textiles has been expanded. Created smart textiles can be used in every-day clothing integrated with electronic elements such as displays, controls, and keyboards enabling e.g. the monitoring of physiological signals [2]. Smart textiles contain e-textiles which due to their electroconductive properties have wide possibilities of applications. They include electrical circuits, transmission lines, antennas, heating elements, electrodes, sensors, EMI shielding materials $[2,4-6,7]$.

The e-textiles are in particular woven fabrics-based. Modeling of the electro-conductive properties of a woven structure is important for designing new textile elements integrated with clothing. Simulation tools make it possible to predict their electro-conductive properties. To determine resistivity, models based on equivalent resistance schemes are used for regular fabrics with yarns being ideal resistors of known resistance $[8,9]$.

The resistivity of woven fabric which is much more complex and shows anisotropy of electrical properties is modeled using the McLachlan equation [10,11]. The McLachlan equation was derived for the conductivity of binary mixtures with anisotropic grain structures [12]. The dependence also works for woven fabrics treated as metal-dielectric composites where conductive linear components (yarns) create a system of empty spaces filled with dielectric air. A prediction level was in the range of $83-88 \%$ for fabrics with surface percentage cover above $96 \%$ [11]. This may be because the contact resistance resulting from the interlaced yarns [7,9] was not taken into account in the study. In the article a new approach to modeling the electro-conductive properties of woven structure was proposed based on a mixing model. The generalized Archie's law developed by Glover $[13,14]$ was chosen. The conductivity of contacts was taken into account in assessing the electro-conductive properties of the woven structure based on the model.

\section{Materials and Methods}

\subsection{Woven structures}

The generalized Archie's law can be applied to objects containing pores filled with phases. The phases are pathways enabling conduction. The remaining part is a non-conducting matrix. The model of the composite was transferred to a woven structure composed of interlaced conductive narrow strips. In the woven structure, the conducting phases are strips and contacts of strips. Due to different conductivities of components, electrical conductivity takes place to varying degrees. The remaining part is pore space filled with dielectric air. It should be emphasized that in the proposed model, the pore space of the woven fabric are not identical with the pores of the model described by generalized law, but oppositely with the nonconducting matrix.

Three woven structures were designed from the same kind of strips. The same dimension $(9 \mathrm{~cm} \times 13 \mathrm{~cm})$ of each structure is assumed. It was assumed that the number of weft and warp stripes is the same i.e. $3 \times 3,4 \times 4$, and $5 \times 5$. The different number of stripes constituting the weft and warp of the woven structure enabled obtaining structures of different densities.

Nine different woven structures were created using strips of $1 \mathrm{~cm}$ wide cut from three different textile materials (woven fabrics). Characteristics of the materials which are important from the point of view of electrical conductivity are given in Table 1.

The linear resistance of $1 \mathrm{~cm}$ wide strips was determined based on the four-electrode method according to EN 16812:2016 standard [15]. The thickness was determined based on EN ISO 5084:1996 standard [16]. Measurements of strip resistance were repeated 10 times, while woven fabric thickness 5 times. The coefficient of variation $\mathrm{CV}$ was given in parentheses. 
Table 1. Characteristics of textile materials used for strips.

\begin{tabular}{lllll}
\hline Woven fabric & $\begin{array}{l}\text { Raw material } \\
\text { composition }\end{array}$ & Manufacturer & $\begin{array}{l}\text { Linear resistance } \\
(\mathbf{\Omega} / \mathbf{c m})\end{array}$ & $\begin{array}{l}\text { Thickness } \\
(\mathbf{m m})\end{array}$ \\
\hline A & $\begin{array}{l}100 \% \text { polyamide woven } \\
\text { fabric; nickel and copper } \\
\text { metalized }\end{array}$ & Laird PLC, GB & $0.035(4 \%)$ & $0.124(4 \%)$ \\
B & $\begin{array}{l}100 \% \text { polyester woven } \\
\text { fabric; nickel metalized }\end{array}$ & Soliani EMC, Italy & $0.180(6 \%)$ & $0.078(6 \%)$ \\
C & $\begin{array}{l}100 \% \text { polyester woven } \\
\text { fabric; nickel metalized }\end{array}$ & Soliani EMC, Italy & $0.246(9 \%)$ & $0.270(4 \%)$ \\
\hline
\end{tabular}

Summarizing, each woven structure was composed of two conducting phases (strips and contacts of strips) and one non-conducting phase (pore space).

\subsection{Mixing model}

Many types of mixing models are used to determine the electrical properties of porous materials. One of them is Archie's law [17] extended for $n$ phases and known as the generalized Archie's law [13]. The Archie's law is empirical quantitative relationship between porosity, electrical conductivity, and brine saturation of rocks [17]. It describes a relationship of the conductivity $\sigma$ of a clean reservoir rock to its porosity $\phi$ and the conductivity of phase $\sigma_{f}$ (e.g. fluid) that completely saturates the pore space. There is also cementation exponent $m$ related to the degree of cementation of the rock fabric. Archie's law can be expressed as follows [13]:

$\sigma=\sigma_{f} \phi^{m}$

where: $\sigma$ - the bulk effective conductivity of the rock; $\sigma_{f}-$ the conductivity of the fluid occupying the pores; $\phi-$ the volume fraction of the fluid phase; $m$ - the cementation exponent.

Small exponent $(m<2)$ occurs for high connectivity phases. Pores that are well connected provide an efficient pathway for the fluid flow. Large exponent $m(m \geq 2)$ occurs for low connectivity phases. The cementation exponent $m$ dependents on the shapes and type of the sedimentary rocks grains, shape and types of pores, specific surface area, tortuosity, anisotropy, and compaction.

Archie's object can be compared to a woven structure composed of two conducting phases (strips and contacts of strips) and one non-conducting phase (pore space). The generalized Archie's law applied to the woven structure can be given as follows [13]:

$\sigma_{\text {str }}=\sigma_{t h} \phi_{t h}^{m_{t h}}+\sigma_{\text {cont }} \phi_{\text {cont }}^{m_{\text {cont }}}$

where: $\sigma_{\text {str }}$ - the woven structure conductivity in $\Omega \mathrm{cm}^{-1} ; \sigma_{t h}, \sigma_{c o n t}-$ the strips and the contacts of strips phases conductivity in $\Omega \mathrm{cm}^{-1}$, respectively; $\phi_{t h}, \phi_{c o n t}-$ the strips and the contacts of strips phases volume fraction, respectively; $m_{t h}, m_{\text {cont }}$ - the exponents of strips and the contacts of strips phases, respectively.

Each of the exponents the generalized law share the same physical meaning as those in the classical Archie's law.

To find the exponents of strips and the contacts of strips phases additional dependence is needed [13]:

$\left(-\frac{\phi_{t h}^{2}}{2}\right) m_{\text {cont }}^{2}+\left(\phi_{t h}-\frac{\phi_{t h}^{2}}{2}\right) m_{\text {cont }}-\phi_{t h}^{m_{t h}}=0$

It was stated that the sum of the volume fractions of all of the phases in porous material must equal unity. The same assumption was adopted for the sum of the connectedness of all of the phases in the porous material.

Based on Eq. 2 and Eq. 3 the exponents of phases can be determined unequivocally. 
The woven structure can be described using two parameters: the connectedness and the connectivity defined by Glover [13] and Glover and Walker [14]. The connectedness $G$ of each phase is given by:

$G_{i}=\frac{\sigma_{s t r}}{\sigma_{i}}$

where: $\sigma_{s t r}-$ the woven structure conductivity in $\Omega \mathrm{cm}^{-1} ; \sigma_{i}-$ the $i$-th phase conductivity in $\Omega \mathrm{cm}^{-1}$.

The connectedness of a given phase is a measure of the availability of pathways for conduction through that phase.

The connectivity $\chi$ for each phase is defined as:

$\chi_{i}=\phi_{i}^{m_{i}-1}$

where: $\phi_{i}-$ the $i$-th phase volume fraction; $m_{i}-$ the $i$-th phase exponent.

The connectivity is a measure of how the components and therefore the pore space is arranged.

\subsection{Measurement methods}

To determine the exponents of strips and the contacts of strips phases, the conductivity of strips, the conductivity of contacts of strips, and the conductivity of woven structure are needed to be identified. The resistance of strip can be determined based on the four-electrode method (Fig. 1a) according to EN 16812:2016 standard [15].

(a)

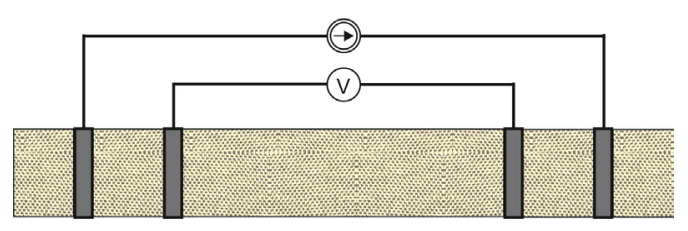

(b)

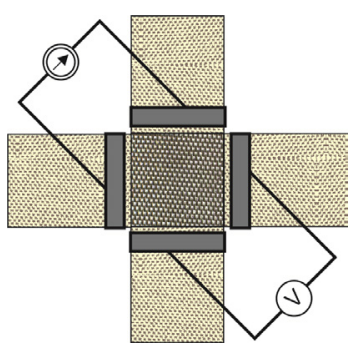

(c)

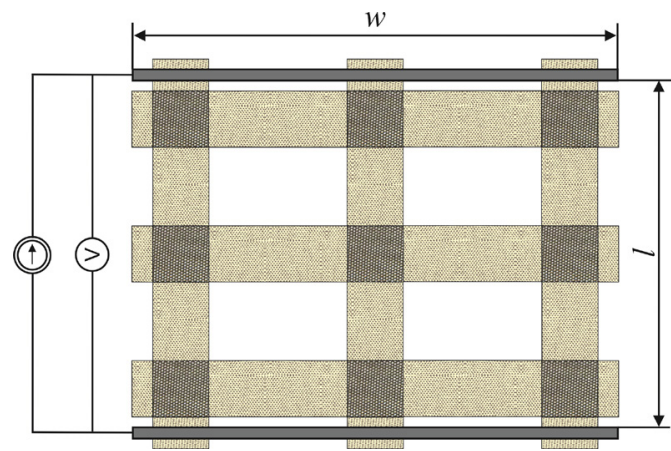

Fig. 1 Resistance measurement (a) Strip; (b) Contact of strips; (c) Woven structure.

The conductivity of strip $\sigma_{\text {th }}$ can be determined based on its linear resistance (Table 1). Therefore the dependence is as follows:

$\sigma_{t h}=\frac{1}{d h R_{L}}$

where: $R_{L}$ - the linear resistance of strip; $d$ - the strip width $(d=1 \mathrm{~cm}) ; h$ - the strip thickness.

The resistance of strips contact can be determined using the four-electrode method (Fig. 1b) described in detail [18]. The conductivity of strips contact $\sigma_{\text {cont }}$ can be calculated from the following dependence:

$\sigma_{\text {cont }}=\frac{1}{2 R h}$

where: $R$ - the resistance of strips contact; $h$ - the strip thickness.

The resistance of woven structure can be performed using two electrodes, four wires method (Fig. 1c) according to AATCC 76-2018 standard [19]. The conductivity of woven structure $\sigma_{\text {str }}$ can be determined using the following formula: 
$\sigma_{s t r}=\frac{l}{w h^{\prime} R}$

wherein

$h^{\prime}=2 h \cdot C_{\text {cont }}+h \cdot C_{t h}$

where: $R$ - the resistance of woven structure; $l-$ the electrodes spacing $(l=9 \mathrm{~cm}) ; w-$ the structure width $(w=13 \mathrm{~cm}) ; h^{\prime}-$ the averaged fabric thickness; $C_{\text {cont }}$ - the fraction of contacts of strips in whole woven structure; $C_{t h}-$ the fraction of strips in the whole woven structure.

\section{Results and Discussion}

Analysis of designed structures was conducted. The fraction $C_{t h}$ and $C_{\text {cont }}$ of strips and contacts of strips, respectively, in the whole structure was determined (Table 2). Remaining part is pores, from which the porosity of the woven structure results.

Table 2. Fraction of strips and contacts of strips in woven structure.

\begin{tabular}{lll}
\hline $\begin{array}{l}\text { Woven structure density } \\
\text { (strips } \times \text { strips) }\end{array}$ & $\begin{array}{l}\text { Fraction of strips } \\
\boldsymbol{C}_{\text {th }}(-)\end{array}$ & $\begin{array}{l}\text { Fraction of contacts of strips } \\
\boldsymbol{C}_{\text {cont }}(-)\end{array}$ \\
\hline $3 \times 3$ & 0.410 & 0.077 \\
$4 \times 4$ & 0.479 & 0.137 \\
$5 \times 5$ & 0.513 & 0.214
\end{tabular}

Based on measurement results and Eq. 6 and Eq. 7, conductivities $\sigma_{\text {th }}$ and $\sigma_{\text {cont }}$ were determined respectively. Based on the values of parameters $C_{t h}$ and $C_{\text {cont }}$ (Table 2) and fabric thickness $h$ (Table 1 ), the averaged fabric thickness $h$ ' was calculated (Eq. 9) for each designed structure. Next, according to Eq. 8, conductivity $\sigma_{\text {str }}$ was determined for each woven structure. Moreover, two phases volume fractions $\phi_{\text {th }}$ and $\phi_{c o n t}$ were determined based on the spacing of strips, the width of strips, and the assumed dimension of the whole woven structure. The sum of the two volume fractions (conductive phases in woven structure) is equal to unity. Solving simultaneous equations (Eq. 2 and Eq. 3), the exponents of phases $m_{\text {th }}$ and $m_{\text {cont }}$ were determined. The received results are juxtaposed in Table 3 . The coefficient of variation was given in parentheses for parameters determined based on measurement results.

Table 3. Parameters of woven structures and their components based on the generalized Archie's law.

\begin{tabular}{llllllll}
\hline $\begin{array}{l}\text { Woven } \\
\text { structure }\end{array}$ & $\begin{array}{l}\sigma_{\text {th }} \\
\left(\boldsymbol{\Omega} \mathbf{c m}^{-1}\right)\end{array}$ & $\begin{array}{l}\sigma_{\text {cont }} \\
\left(\mathbf{\Omega c m}^{-1}\right)\end{array}$ & $\begin{array}{l}\sigma_{\text {str }} \\
\left(\boldsymbol{\Omega c m}^{-1}\right)\end{array}$ & $\begin{array}{l}\phi_{\text {th }} \\
(-)\end{array}$ & $\begin{array}{l}\phi_{\text {cont }} \\
(-)\end{array}$ & $\begin{array}{l}\boldsymbol{m}_{\text {th }} \\
(-)\end{array}$ & $\begin{array}{l}\boldsymbol{m}_{\text {cont }} \\
(-)\end{array}$ \\
\hline $3 \times 3$ A & $2291(4 \%)$ & $3200(17 \%)$ & $1032(7 \%)$ & 0.84 & 0.16 & 4.7 & 3.0 \\
$3 \times 3$ B & $712(6 \%)$ & $3339(13 \%)$ & $302(2 \%)$ & 0.84 & 0.16 & 5.2 & 3.0 \\
$3 \times 3$ C & $150(9 \%)$ & $146(11 \%)$ & $69(1 \%)$ & 0.84 & 0.16 & 4.5 & 2.9 \\
$4 \times 4$ A & $2291(4 \%)$ & $3200(17 \%)$ & $967(4 \%)$ & 0.78 & 0.22 & 3.6 & 3.1 \\
$4 \times 4$ B & $712(6 \%)$ & $3339(13 \%)$ & $286(7 \%)$ & 0.78 & 0.22 & 4.0 & 3.2 \\
$4 \times 4$ C & $150(9 \%)$ & $146(11 \%)$ & $70(9 \%)$ & 0.78 & 0.22 & 3.1 & 3.1 \\
$5 \times 5$ A & $2291(4 \%)$ & $3200(17 \%)$ & $911(5 \%)$ & 0.71 & 0.29 & 2.8 & 3.4 \\
$5 \times 5$ B & $712(6 \%)$ & $3339(13 \%)$ & $286(7 \%)$ & 0.71 & 0.29 & 3.2 & 3.4 \\
$5 \times 5$ C & $150(9 \%)$ & $146(11 \%)$ & $68(4 \%)$ & 0.71 & 0.29 & 2.4 & 3.3 \\
\hline
\end{tabular}

Pearson's correlation coefficient $R_{P}$ was used in statistics to measure how strong a relationship is between the conductivity of strips, contacts of strips, and whole woven structure. The significance level equals 0.10 was assumed. It was noticed that for couples $\sigma_{t h}, \sigma_{c o n t}$, and $\sigma_{c o n t}, \sigma_{s t r}$, a strong uphill (positive) linear relationship is observed; $R_{P}=0.675$ and $R_{P}=0.661$ were received respectively. For a couple of $\sigma_{\text {th }}, \sigma_{\text {str }}$, an almost perfect uphill (positive) linear relationship was noticed $\left(R_{P}=0.997\right)$. This is due to the fact that the strips phase volume fraction $\phi_{t h}$ this greater than the contacts of strips phase volume fraction $\phi_{c o n t}$. 
Relatively small values of $\phi_{c o n t}$ and low level of diversity of woven structures were reflected in the comparable values of phase exponent $m_{\text {cont. }}$. For all woven structures the average value of the parameter equals 3.16 , whereas $C V$ is equal to $6 \%$. Values of $m_{\text {th }}$ were considered in groups divided by structure density. Therefore following results were obtained for structures: $3 \times 3, m_{\text {th }}=4.84, C V=8 \% ; 4 \times 4, m_{\text {th }}=3.58$, $C V=12 \% ; 5 \times 5, m_{t h}=2.79, C V=14 \%$. In addition to structure features, the exponents also depend indirectly on the conductivity of individual components. This is due to the mixing model (Eq. 2). It can be generally stated that the even woven structure (see Fig. 1c), where identical pores in the shape of rectangles and conductive phases are evenly distributed throughout the structure, characterizes large values of phase exponent (above 2). Therefore the low connectivity of conductive phases occurs. The smaller the woven structure density, the larger the phase exponent $m_{t h}$.

Next, the connectedness $G$ (Eq. 4 ) and the connectivity $\chi$ (Eq. 5) for each phase were calculated and given in Table 4.

Table 4. Parameters describing electro-conductive woven structure.

\begin{tabular}{lllll}
\hline Woven structure & $\boldsymbol{G}_{\text {th }}(-)$ & $\boldsymbol{G}_{\text {cont }}(-)$ & $\chi_{\text {th }}(-)$ & $\chi_{\text {cont }}(-)$ \\
\hline $3 \times 3 \mathrm{~A}$ & 0.45 & 0.32 & 0.53 & 0.03 \\
$3 \times 3 \mathrm{~B}$ & 0.42 & 0.09 & 0.48 & 0.02 \\
$3 \times 3 \mathrm{C}$ & 0.46 & 0.48 & 0.54 & 0.03 \\
$4 \times 4 \mathrm{~A}$ & 0.42 & 0.30 & 0.53 & 0.04 \\
$4 \times 4 \mathrm{~B}$ & 0.40 & 0.09 & 0.47 & 0.04 \\
$4 \times 4 \mathrm{C}$ & 0.46 & 0.48 & 0.58 & 0.04 \\
$5 \times 5 \mathrm{~A}$ & 0.40 & 0.28 & 0.53 & 0.05 \\
$5 \times 5 \mathrm{~B}$ & 0.40 & 0.09 & 0.47 & 0.05 \\
$5 \times 5 \mathrm{C}$ & 0.45 & 0.47 & 0.62 & 0.06 \\
\hline
\end{tabular}

The connectedness of the strips phase and the contacts of strips phase indicates the availability of the pathways for conduction through this phase. Therefore it is a qualitative feature. The conductivity of each phase was higher than the conductivity of the woven structure therefore values of $G$ are in the range of $(0,1)$. It was noticed, that adding another component to the woven structure can reduce the conductivity of the structure, which results in a decrease of parameter $G$ (Table 4).

It was found, that the connectedness of both phases slightly increases with decreasing woven structure density. It is particularly visible for both phases of woven structures designed using strips of the woven fabric denoted as A (Fig. 2a). The extent to which the strips phase and the contacts of strips phase affect (in terms of their quality) the conductivity of the woven structure depends on woven fabric. In most cases, the impact of the strips phase is greater than the contacts of strips phase as shown in Fig. $2 a$.

(a)

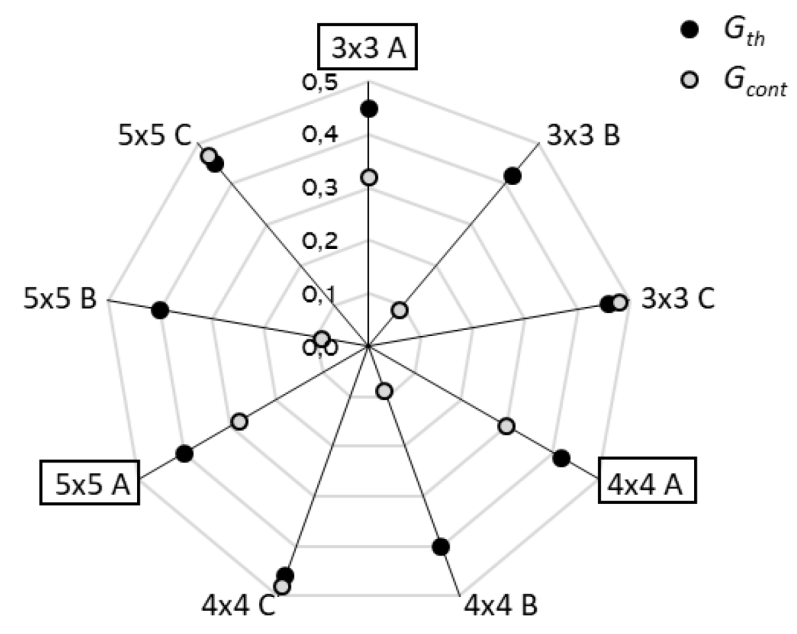

(b)

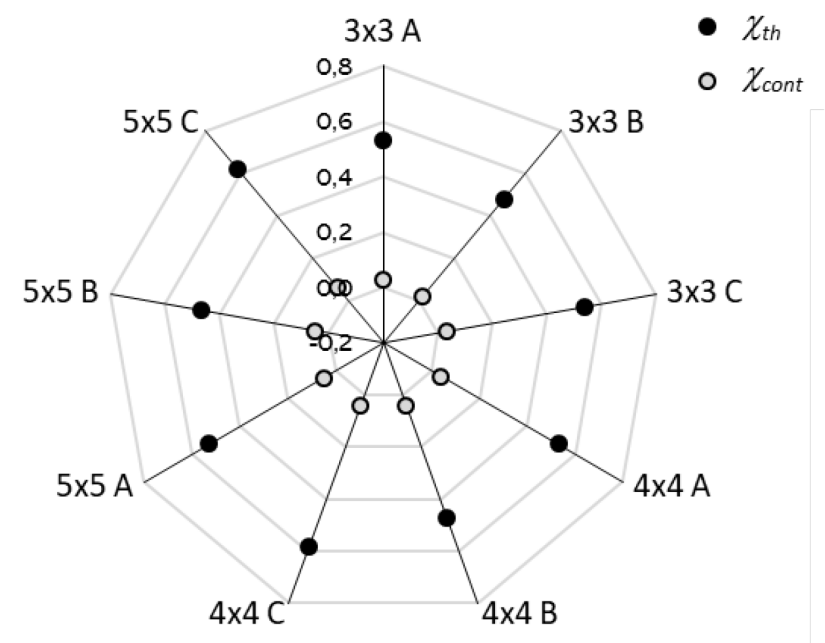

Fig. 2 Parameters of strips and contacts of strips phases (a) Connectedness; (b) Connectivity. 
The connectivity indicates how components are arranged in a woven structure. The values of connectivity $\chi$ are greater than 0 . The effect of strips phase and contacts of strips phase on the woven structure conductivity are presented in Fig. 2b.

The larger values of $\chi_{\text {th }}$ compared to $\chi_{\text {cont }}$ mean that the strips phase (in terms of their quantity) has a greater effect on the conductivity of the woven structure than the contacts of strips phase. It was observed for all woven structures. However, it should be noted, this parameter is related to phase exponent which indirectly implies the impact of the components' conductivity on the connectivity value.

\section{Conclusions}

It was found, that the chosen mixing model can be used to modeling the electro-conductive properties of woven structure which consists of electro-conductive components i.e. strips and contacts of strips treated as conducting phases in the whole structure.

A strong uphill linear relationship was observed for conductivities of strips and contacts of strips phases, and also for conductivities of contacts of strips and woven structure. An almost perfect uphill linear relationship was observed for conductivities of strips and woven structure.

A decrease of the connectedness of strips and contacts of strips phases (in terms of their quality) can be obtained by adding another component to the woven structure which will reduce the conductivity of the whole structure.

The larger values of connectivity $\chi_{\text {th }}$ compared to $\chi_{\text {cont }}$ mean that the strips phase (in terms of their quantity) has a greater effect on the conductivity of the woven structure than the contacts of strips phase. However, contacts of strips must be taken into account in assessing the electro-conductive properties of the woven structure. The generalized Archie's law enables such analysis and from this point of view, it is better than the McLachlan model.

By designing the same structure but from other components, you can predict the conductivity of the new structure based on parameters determined from the mixing model. It was found that the phase exponent $m_{\text {cont }}$ equals 3.16 for all woven structures and the exponent $m_{\text {th }}$ equals $2.79,3.58$, and 4.84 for structures $5 \times 5,4 \times 4$, and $3 \times 3$, respectively. The smaller the woven structure density, the larger the phase exponent $m_{\text {th }}$. Having a well-defined electrical conductivity, volume fraction, and exponent for all phases, the electrical conductivity of the whole composite can be calculated and compared to the experimental result.

\section{References}

[1] Cherenack, K.; van Pieterson, L. Smart textiles: Challenges and opportunities. J App/ Phys, 2012, 112(9), 091301-1-091301-14. DOI: 10.1063/1.4742728.

[2] Gonçalves, C.; Ferreira da Silva, A.; Gomes, J.; Simoes, R. Wearable e-textile technologies: A review on sensors, actuators and control elements. Inventions, 2018, 3(1), 1-13. DOI: 10.3390/inventions3010014.

[3] Castano, L.M.; Flatau, A.B. Smart fabric sensors and e-textile technologies: A review. Smart Mater Struct, 2014, 23, 053001-053027. DOI: 10.1088/0964-1726/23/5/053001.

[4] Acar, G.; Ozturk, O.; Golparvar, A.J.; Elboshra, T.A.; Böhringer, K.; Yapici, M.K. Wearable and flexible textile electrodes for biopotential signal monitoring: A review. Electronics, 2019, 8(5), 1-25. DOI: 10.3390/electronics8050479.

[5] Gil, I.; Fernández-García, R.; Tornero, J.A. Embroidery manufacturing techniques for textile dipole antenna applied to wireless body area network. Text Res J, 2019, 89(8), 1573-1581. DOI: 10.1177/0040517518770682.

[6] Shaw, V.P.; Jagatheesan, K.; Ramasamy, A. Mechanical and electromagnetic shielding behaviours of thermoplastic conductive composite: Influence of yarn structure and process variables. J Text I, 2019. DOI: $10.1080 / 00405000.2019 .1688903$.

[7] Zeng, W.; Shu, L.; Li, Q.; Chen, S.; Wang, F.; Tao, X-M. Fiber-based wearable electronics: A review of materials, fabrication, devices, and applications. Adv Mater, 2014, 26, 5310-5336. DOI: 10.1002/adma.201400633.

[8] Liu, S.; Tong, J.; Yang, C.; Li, L. Smart e-textile: Resistance properties of conductive knitted fabric - Single pique. Text Res J, 2017, 87(14), 1669-1684. DOI: 10.1177/0040517516658509.

[9] Gunnarsson, E.; Karlsteen, M; Berglin, L; Stray, J. A novel technique for direct measurements of contact resistance between interlaced conductive yarns in a plain weave. Text Res J, 2015, 85(5), 499-511. DOI: $10.1177 / 0040517514532158$. 
[10] Tokarska, M. New concept in assessing compactness of woven structure in terms of its resistivity. $J$ Mater SciMater El, 2016, 27(7), 7335-7341. DOI: 10.1007/s10854-016-4703-2.

[11] Tokarska M., Mathematical model for predicting the resistivity of an electroconductive woven structure. J Electron Mater, 2017, 46(3), 1497-1503. DOI: 10.1007/s11664-016-5186-X.

[12] McLachlan, D.S. Equations for the conductivity of macroscopic mixtures. J Phys C Solid State, 1986, 19(9), 1339-1354. DOI: 10.1088/0022-3719/19/9/007.

[13] Glover, P.W.J. A generalized Archie's law for $n$ phases. Geophysics, 2010, 75(6), E247-E265. DOI: $10.1190 / 1.3509781$.

[14] Glover, P.W.J.; Walker, E. Grain-size to effective pore-size transformation derived from an electrokinetic theory. Geophysics, 2009, 74(1), E17-E29. DOI: 10.1190/1.3033217.

[15] EN 16812:2016. Textiles and textile products. Electrically conductive textiles. Determination of the linear electrical resistance of conductive tracks. 2016.

[16] EN ISO 5084:1996. Textiles. Determination of thickness of textiles and textile products. 1996.

[17] Archie, G.E. Electrical resistivity log as an aid in determining some reservoir characteristics. T Metall Soc AIME, 1942, 146(1), 54-62. DOI: 10.2118/942054-G.

[18] Vasile, S.; Deruck, F.; Hertleer, C.; De Raeve, A.; Ellegiers, T.; De Mey, G. Study of the contact resistance of interlaced stainless steel yarns embedded in hybrid woven fabrics. Autex Res J, 2017, 17(2), 170-176. DOI: 10.1515/aut-2016-0024.

[19] AATCC 76-2018. Electrical resistivity of fabrics. 2018. 